\title{
Misdiagnosis of laryngeal sarcomatoid squamous cell carcinoma - case report
}

\author{
Irén Csiszér ${ }^{1,2}$, Simona Mocanu ${ }^{3}$, Vlad Budu ${ }^{4}$, Adriana Neagos ${ }^{5}$
}

${ }^{1}$ Department of Otorhinolaryngology, Emergency County Hospital of Targu Mures, Romania

${ }^{2}$ Department of Otorhinolaryngology, "Iuliu Hatieganu” University of Medicine and Pharmacy, ClujNapoca, Romania

${ }^{3}$ Department of Pathologic Anatomy, Emergency County Hospital of Targu Mures, Romania ${ }^{4}$ Department of Otorhinolaryngology, "Carol Davila” University of Medicine and Pharmacy, Bucharest

${ }^{5}$ Department of Otorhinolaryngology, University of Medicine and Pharmacy of Targu Mures, Romania

\section{ABSTRACT}

BACKGROUND. Even if it is a rare variant growth pattern of squamous cell carcinoma, sarcomatoid carcinoma seems to have almost the same clinical manifestations and risk factors as conventional squamous cell carcinoma.

CASE REPORT. This paper presents a complex case of a 59-year-old female patient known with laryngeal papillomatosis, who presented in our Department for moderate to severe inspiratory dyspnea associated with dysphonia and difficulty in swallowing. We mention that the patient had been previously evaluated by a pneumologist for apnea, snoring and daytime sleepiness and diagnosed and treated for sleep apnea syndrome. Clinical, laboratory, imaging and pathologic examinations revealed the association of upper airway obstruction with laryngeal cancer. Surgical intervention consisted in total laryngectomy and selective neck dissection associated with permanent tracheotomy. The microscopic appearance was that of a bilateral transglottic sarcomatoid squamous cell carcinoma without metastasis in the right lymph node.

CONCLUSION. It is important to evaluate the clinical and imagistic status of patients with laryngeal tumors in order to make a correct decision concerning their treatment policy. We emphasize that cooperation between multiple departments is absolutely necessary in order to adequately resolve, explore, diagnose and treat patients with laryngeal pathology.

KEYWORDS: obstructive sleep apnea syndrome, sarcomatoid carcinoma, laryngeal papillomatosis.

\section{INTRODUCTION}

Sarcomatoid carcinoma of the larynx is a rare form of squamous cell carcinoma, representing almost $2 \%$ of these tumors ${ }^{1-3}$. The squamous cell carcinoma is the most frequent type of laryngeal cancer, comprising more than $90 \%$ of all head and neck malignancies ${ }^{4}$. It is predominantly diagnosed in male patients, in the $6^{\text {th }}$ decade of life, the literature reporting a mean age of 65.6 years ${ }^{1,3,5-7}$.

The occurrence of laryngeal carcinoma is most of the time related to alcohol or tabacco use, occupational factors, air pollution or gastroesophageal reflux disease, Human Papilloma Virus infection ${ }^{3,7,8}$. There are also cases in which a benign lesion, such as laryngeal papillomatosis, turns into a malignant one $\mathrm{e}^{9-11}$.
The primary symptom is dysphonia, accompanied in early stages by other nonspecific laryngeal symptoms, such as persistent caught, or feeling that something is stuck in the neck. In advanced stages, inspiratory dyspnea, daytime sleepiness, difficult swallowing or stridor may be observed.

The aim of this report was to draw attention upon the importance of a complete evaluation of a patient with known laryngeal pathology, to present the malignant transition of the laryngeal papillomatosis and nevertheless to underline, once again if necessary, that the multidisciplinary approach is essential in patients with upper respiratory tract diseases. 


\section{CASE REPORT}

We present the case of a 59-year-old female patient, who presented to the ENT Clinic of Targu Mures Emergency County Hospital in 2011 for moderate to severe inspiratory dyspnea associated with dysphonia and difficulty in swallowing, symptoms aggravated after an acute upper airways infection. During the anamnesis the patient reported that she had been previously evaluated by a pneumologist for apnea, snoring and daytime sleepiness and diagnosed and treated for sleep apnea syndrome. What drew our attention was that the patient has been diagnosed in 1997 with laryngeal papillomatosis.

Between 1997 and 2000 she underwent 4 surgical interventions. The anatomopathological exam performed in 2000 revealed a malignant transformation of the laryngeal papillomatosis - microinvasive epidermoid carcinoma, stage T2N0M0. The cervical CT-scan performed at that moment revealed a minor asymmetry of the vocal cords due to a protruding mass with a nodular appearance situated on the left vocal cord, and an asymmetry at the level of the right ventricular band.

Considering the new diagnosis, the patient was referred for radiotherapy, a total dose of 66Gy in 33 fractions for 43 days. The therapy was well tolerated, with a tumor regression to T0N0Mx. Between 2000 and 2010 our patient was followed-up periodically by the ENT specialist and the oncologist.

Also, from the patient personal pathological history we noted: type II non-controlled diabetes mellitus, chronic viral hepatitis $\mathrm{C}$, hypothyroidism, chronic ischemic cardiomyopathy with prior myocardial infarction sequelae, cerebral stroke sequelae.

The clinical otorhinolaryngological and general examination performed at the admittance in our Department in December 2011 revealed an altered general state, cold and sweaty skin. The indirect laryngoscopy and the nasopharyngeal fibroscopy showed a vegetating nodular lesion whose point of origin was the left vocal cord, immobilizing the left hemilaryngeal part, a mobile right vocal cord, a reduced glottis space and an edematous hypopharyngeal wall. The cervical CT-scan showed a left paramedian infraglottic space-occupying mass measuring approximately 15x29 mm (Figure 1), with precise outlines, with median destruction extending towards the posterior hypopharyngeal wall and an adenopathy measuring $6 \mathrm{~mm}$, localized on the anterior cervical chain.

The decision was to perform a supraisthmic tracheotomy and direct suspension laryngoscopy with biopsy. The histopathological report revealed that the tumor was a sarcomatoid carcinoma.

Considering patient's comorbidities, a multidisciplinary evaluation (cardiologic, infectious, laboratory, pneumologic, diabetologic, neurologic and endocrinologic) was carried out in order to evaluate the patient's clinical status.

Considering the histopathological results, the indication was for a total laryngectomy. Because it was difficult for the patient to accept due to its psychosocial impact as well as side effects that were hard to cope with, the intervention was performed in March 2012.

At that time, a selective neck dissection associated with permanent tracheotomy was performed. The histopathological findings showed a bilateral transglottic sarcomatoid squamous cell carcinoma, without lymph node metastases; clean surgical resection edges pT3N0Mx; microscopic description (Figure 2) showing an infiltrative nodular proliferation at the transglottic laryngeal level, consisting of elongated, spindleshaped tumor cells with rounded ends arranged in long intersecting strands with hyperchromatic, pleomorphic nuclei and several atypical mitotic figures. The tumor surface was almost completely ulcerated.

The thickness of the tumor invasion was $11 \mathrm{~mm}$. The desmoplastic reaction and the peritumoral chronic inflammatory infiltration were moderate. There were no noticeable lymphovascular emboli, but perineural invasions were present. There were no metastases noticed in the laterocervical lymph node samples and the surgical resection edges were clean. The tumor cells showed positivity for SMA, and the reactions to pancytokeratin, EMA, Desmin, S-100 and p53 were negative. The pathologist did not consider negativity to pan-Cytokeratin (AE1 and AE3) to be an argument for a different diagnosis than sarcomatoid carcinoma since he believed that tumor cells may have re-

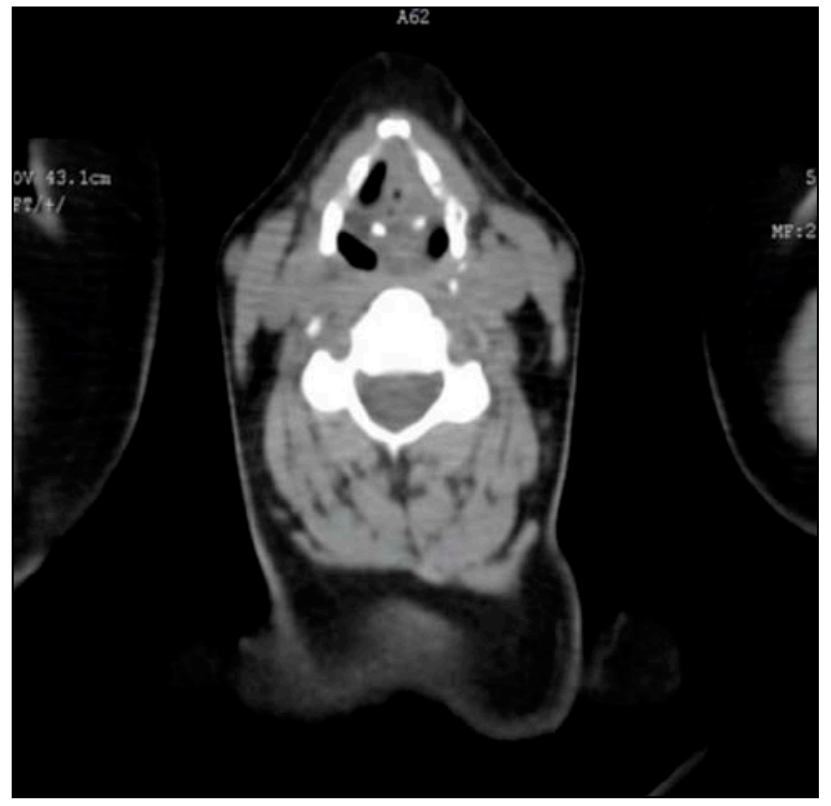

Figure 1 Cervical CT-scan, axial slice - left paramedian infraglottic spaceoccupying mass measuring approximately $15 \times 29 \mathrm{~mm}$ 


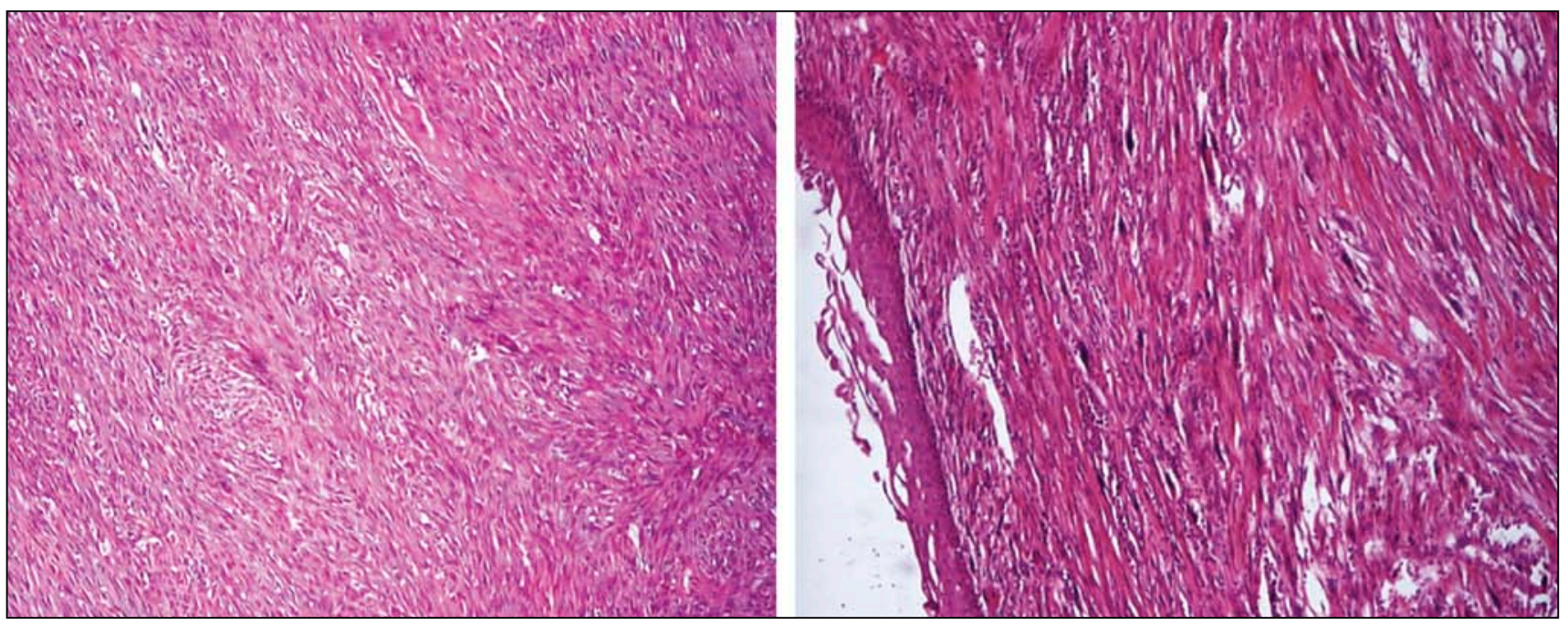

Figure 2 Histopathologic exam - elongated, spindle-shaped tumor cells with rounded ends arranged in long intersecting strands with hyperchromatic, pleomorphic nuclei and several atypical mitotic figures.
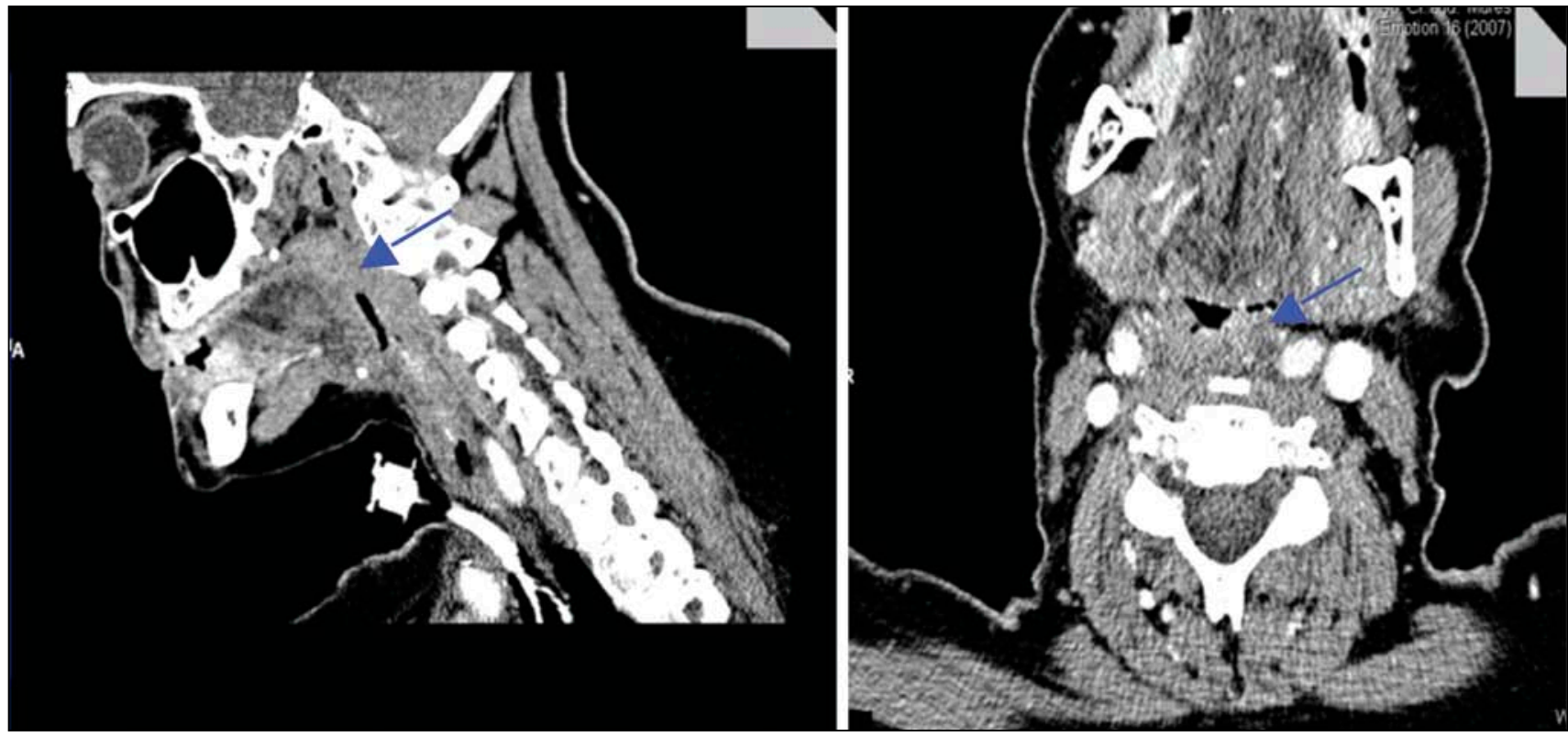

Figure 3 Cervical CT-scan, sagittal and axial slices - space-occupying mass located at the level of the hypopharynx, protruding from the left posterolateral outline, measuring 14/10/16 $\mathrm{mm}$ and producing a marked asymmetry of the lumen, with bilateral laterocervical adenopathies measuring $8 \mathrm{~mm}$.

acted negatively to this marker due to fixation and staining, which could cause phenotypic alterations.

The immediate postoperative outcome was favourable and the patient received chemotherapy treatment postoperatively. 6 months after the surgical procedure the patient returned to our department complaining of difficulty in swallowing, quasi-total dysphagia with recent onset (approximately 3 days) with an extremely fast progression, and micro-hemorrhages externalized through the oral cavity. The ENT examination and the flexible nasopharyngeal fibroscopy raised the suspicion of a hemorrhaging tumor relapse, for which a cervical tomography was repeated. The investigation revealed the presence of a space-occupying mass located at the level of the hypopharynx, protruding from the left posterolateral outline, measuring 14/10/16 $\mathrm{mm}$ and producing a marked asymmetry of the lumen (Figure 3), with bilateral laterocervical adenopathies measuring $8 \mathrm{~mm}$.

In light of the examinations that matched a tumor relapse, the decision was to perform direct suspension laryngoscopy for diagnostic purposes and a bioptic sample excision treatment at the level of the hypopharyngeal tumor mass, as well as to reduce the tumor size by excision and cauterization. The histopathological findings from the bioptic sample taken showed a tumor relapse histopathologically similar to the tumor excised 6 months before.

Unfortunately, at the moment of writing this report the patient had died. 


\section{DISCUSSIONS}

Even if it is a rare variant growth pattern of squamous cell carcinoma, sarcomatoid carcinoma seems to have almost the same clinical manifestations and risk factors as conventional squamous cell carcinoma. In this particular type of laryngeal neoplasm, the literature suggests that radiation therapy can be considered an etiological factor, the latency period being described between 1.2 to 16 years from the radiation exposure $^{3,5,7,11-13}$. In our case the patient underwent 43 days of radiotherapy 11 years prior to the development of the sarcomatoid carcinoma.

This present clinical case is important because the apnea and the daytime sleepiness, for which the patient was first evaluated by the pneumologist, were produced by a laryngeal malignant tumor. Very rarely, but the tumoral pathology can be the etiology of sleep disordered breathing. It is important to establish the real cause of sleep problems, before saying that it is or not an obstructive sleep apnea.

It is very well known that obstructive sleep apnea syndrome is a common sleep-disordered breathing, characterized by recurrent episodes of upper airway obstruction and a significant decrease (hypopnea) or cessation (apnea) in airflow in the presence of thoracoabdominal breathing movements ${ }^{14-18}$. Each episode lasts at least 10 seconds $^{14,17,19-21}$. The implication of craniofacial anomalies, laryngeal morphology and pathology is very important in the evaluation of patients with OSAS ${ }^{15,22-25}$. The diagnosis of obstructive sleep apnea is based on otorhinolaryngological examination, nasopharyngeal endoscopy / nasolaryngoscopy, imagistic examinations, polysomnography with a minimum of 7 channels and a multidisciplinary evaluation (ENT, pneumologist, cardiologist, dentist and neurologist). So, the obtained data must be correlated and analyzed in order to identify the cause of the symptoms and then the cause of the apnea must be treated with high efficiency ${ }^{17,20,23,24}$.

In our case, not the sleep apnea was the important factor for daytime sleepiness, but the dyspnea. It would have been important to take into consideration the pathologic history of the patient and to make an early evaluation of the larynx. During the 15-year period since the initial diagnosis, repeated histopathological examinations showed relapsed laryngeal papillomatosis that in 3 years underwent malignization for which the patient had received radiation treatment.

It is essential to diagnose the laryngeal cancer in early stages when the survival rate can be higher than $80 \%{ }^{4,26}$. Five-year survival rate is reported as being of $60 \%$.

The swift progression of the disease, in our case, required major therapeutic measures such as emergency tracheotomy, total laryngectomy, treatments that were difficult for the patient to accept because of their psychosocial impact, as well as side effects that were hard to cope with. Although the disease had not caused any particular problems in 10 years' time, the outcome was highly unfavourable despite the fact that the emergent tumor was surgically dealt with in an adequate stage, excised within safe limits, and did not metastasize.

The clean surgical edges in the case of the sarcomatoid carcinoma in a patient with reduced immunity are not a prognosis factor that can guarantee the absence of a tumor relapse, as it can be seen in our patient.

Given this case, we have become determined to follow-up more closely and request additional testing to monitor the patients with laryngeal papillomatosis. Also, it is important to evaluate the extension and the histopathologic type of the tumor in correlation with the clinical status of the patient, the age and the risk factors $^{27,28}$.

\section{CONCLUSIONS}

Considering the case presented, we believe that it is important to evaluate the clinical and imagistic status of patients with laryngeal tumors in order to make a correct decision concerning their treatment policy.

Since patients with sleeping disorders end up at a neurologist, a psychiatrist or a pneumologist where they are treated symptomatically, as it was in this case, we believe that it is very important for them to also have a complete otorhinolaryngological evaluation in order to exclude diseases affecting the upper respiratory tract.

In case of tumors, repeated biopsies do not exclude malignization, and thus are no guarantee that a benign mass will not become malignant.

As a consequence, we emphasize that cooperation between multiple departments is absolutely necessary in order to adequately resolve, explore, diagnose and treat patients with laryngeal pathology.

\section{REFERENCES}

1. Brodsky G. - Carcino(pseudo) sarcoma of the larynx: the controversy continues. Otolaryngol Clin North Am., 1984;17:185-97.

2. Ferlito A. - Histological classification of larynx and hypopharynx cancers and their clinical implications: pathologic aspects of 2052 malignant neoplasm diagnosed at the ORL Department of Padua University from 1966 to 1976. Acta Otolaryngol Suppl., 1976;342:1-88.

3. Thompson L.D.R., Weineke J.A., Miettinen M., Heffner D.K. - Spindle Cell (Sracomatoid) Carcinomas of the Larynx: A Clinicopathologic Study of 187 Cases. Am J Surg Pathol., 2002;26(2):153-170.

4. Latifi H., Mikaili P., Latifi K., Torbati H. - Squamous cell carcinoma of larynx in northwestern Iran. Euro J Exp Bio., 2012;2(1):242-246.

5. Hellquist H., Olofsson J. - Spindle cell carcinoma of the larynx. 
APMIS, 1989;97:1103-13.

6. Lambert P.R., Ward P.H., Berci G. - Pseudosarcoma of the larynx: a comprehensive analysis. Arch Otolaryncol., 1980;106:700-8.

7. Viswanathan S., Rahman K., Pallavi S., Sachin J., Patil A., Chaturvedi P., D’Cruz A., Agarwal J., Kane S.V. - Sarcomatoid (Spindle Cell) Carcinoma of the Head and Neck Mucosal Region: A Clinicopathologic Review of 103 Cases from Tertiary Referral Cancer Centre. Head and Neck Pathol., 2010;4:265-175.

8. Rua S., Comino A., Fruttero A., Cera G., Semeria C., Lanzillotta L., Boffetta P. - Relationship between histologic features, DNA flow cytometry, and clinical behavior of squamous cell carcinomas of the larynx. Cancer, 1991;67:141-149.

9. Gillison M.L., Castellsague X., Chaturvedi A., Goodman M.T., Snijders P., et al. - Eurogin Roadmap: comparative epidemiology of HPV infection and associated cancers of the head and neck and cervix. Int J Cancer, 2014;134:497-507. doi: 10.1002/ijc.28201.

10. Hobbs C.G., Birchall M.A. - Human papilloma virus infection in the etiology of laryngeal carcinoma. Curr Opin Otolaryngol Head Neck Surg., 2004;12:88-92. doi: 10.1097/00020840-200404000-00006.

11. Olsen K.D., Lewis J.E., Suman V.J. - Spindle cell carcinoma of the larynx and hypopharynx. Otolaryngol Head Neck Surg., 1997;116:4752.

12. Ellis G.L., Corio R.L. - Spindle cell carcinoma of the oral cavity: a clinicopathologic assessment of fifty-nine cases. Oral Surg Oral Med Oral Pthol., 1980;50:523-33.

13. Lewis J.E., Olsen K.D., Sebo T.J. - Spindle cell carcinoma of the larynx: review of 26 cases including DNA content and immunohistochemistry. Hum Pathol., 1997;28:664-73.

14. Todea A.D., Albu S., Ariesanu N. et al. - Apeea in somn si comorbiditatile sale. Editura Medicala Universitara "Iuliu Hatieganu”, Cluj Napoca, 2011;pp.9-37;73-103.

15. Kato T., Mikami A., Sugita H., Muraki H., Okura M., Ohi M., Taniguchi M. - Negative association between self-reported jaw symptoms and apnea-hypopnea index in patients with symptoms of obstructive sleep apnea syndrome: a pilot study. Sleep Breath., 2013;17(1):373379. doi: 10.1007/s11325-012-0704-4. Epub 2012 Apr 18.

16. Schraff S., Derkay C.S., Burke B., Lawson L. - American Society of Pediatric Otolaryngology members' experience with recurrent respiratory papillomatosis and the use of adjuvant therapy. Arch Otolaryngol Head and Neck Surg., 2004;130(9):1039-42.

17. Hargens T., Kaleth A., Edwards E., Butner K. - Association between sleep disorders, obesity, and exercise: a review. Nat Sci Sleep, 2013;5:27-35. doi: 10.2147/NSS.S34838. Print 2013.
18. Mannarino M., Di Filippo F., Pirro M. - Obstructive sleep apnea syndrome. Eur J Intern Med., 2012;23(7):586-593. doi: 10.1016/j. ejim.2012.05.013. Epub 2012 Jun 24.

19. Yano Y., Ohmori T., Shimada K., Sakata Y., Kario K. - Association of sleep onset of acute coronary syndrome with sleep apnea syndrome and abnormal diurnal variation of hemostasis and adipokine levels. Blood Coagul Fibrinolysis, 2012;23(7):590-596.

20. Karkoulias K., Lykouras D., Sampsonas F., Karaivazoglou K., Sargianou M., Drakatos P., Spiropoulos K., Assimakopoulos K. - The impact of obstructive sleep apnea syndrome severity on physical performance and mental health. The use of SF-36 questionnaire in sleep apnea. Eur Rev Med Pharmacol Sci., 2013;17(4):531-536.

21. Carlucci M., Smith M., Corbridge S.J. - Poor sleep, hazardous breathing: An overview of obstructive sleep apnea. Nurse Pract., 2013;38(3):20-28, quiz 28-9. doi: 10.1097/01.NPR.0000426409.62476. fa.

22. Fidan F., Özbulut Ö., Ayçiçek A., Așik A., Ünlü M. - Effects of obstructive sleep apnea syndrome on temperament and character. Turk J Med Sci., 2013;43(1):138-143.

23. Assoumou H., Gaspoz J., Sforza E., Pichot V., Celle S., et al. Obstructive sleep apnea and the metabolic syndrome in an elderly healthy population: the SYNAPSE cohort. Sleep Breath., 2012;16(3):895-902. doi: 10.1007/s11325-011-0593-y. Epub 2011 Sept 18.

24. Asghari A., Mohammadi F., Kamrava S., Jalessi M., Farhadi M. Evaluation of quality of life in patients with obstructive sleep apnea. Eur Arch Otorhinolaryng., 2013;270(3):1131-1136. doi: 10.1007/ s00405-012-2157-6. Epub 2012 Aug 19.

25. Iosif C. - Notiuni de histologie si morfologie ale laringelui. In: Sarafoleanu C. (edit) - Laringologie. Editura Academiei Romane, Bucuresti, 2007;pp.135-158.

26. Olsen J., Sabroe S., Fasting U. - Interaction of alcohol and tobacco as risk factors of cancer of the laryngeal region. J Epidemiol Community Health, 1985;39:165-8.

27. Sarafoleanu C. - Tumorile benigne ale laringelui. In: sub red. Popescu I., Ciuce C., coord. Sarafoleanu C. - Tratat de chirurgie. Vol. 1: Otorinolaringologie si chirurgie cervico-faciala. Editura Academiei Romane, Bucuresti, 2012;p.199-217.

28. Sarafoleanu C., Postelnicu V. - Patologia tumorala maligna a laringelui. In: sub red. Popescu I., Ciuce C., coord. Sarafoleanu C. - Tratat de chirurgie. Vol. 1: Otorinolaringologie si chirurgie cervico-faciala. Editura Academiei Romane, Bucuresti, 2012;p.277-314. 